\title{
Prevalência do uso de drogas antidepressivas por estudantes da área da saúde no
}

\author{
Brasil \\ Prevalence of antidepressant drug use by health students in Brazil \\ Prevalencia del consumo de drogas antidepresivas por estudiantes de salud en Brasil
}

Recebido: 20/08/2021 | Revisado: 25/08/2021 | Aceito: 30/08/2021 | Publicado: 01/09/2021

Kaisy Alves de Oliveira

ORCID: https://orcid.org/0000-0001-9778-8938 Faculdade de Ciências Médicas da Paraíba, Brasil

E-mail: kaisyalves7@gmail.com

George Estrela de Oliveira

ORCID: https://orcid.org/0000-0003-3331-4228 Faculdade de Ciências Médicas da Paraíba, Brasil E-mail: george_estrela@yahoo.com.br

Claudine Kênnia Almeida Cezário

ORCID: https://orcid.org/0000-0002-5765-3630 Faculdade de Ciências Médicas da Paraíba, Brasil E-mail: claudine.nutri@gmail.com

Mayrlla Myrelly Vieira Formiga ORCID: https://orcid.org/0000-0001-8962-6242 Faculdade de Ciências Médicas da Paraíba, Brasil E-mail: mayrlla.myrelly15@gmail.com

Andrea Paloma Ferreira de Siqueira

ORCID: https://orcid.org/0000-0003-0095-0033

Faculdade de Ciências Médicas da Paraíba, Brasil E-mail: andreasiqueiramt@gmail.com

Ana Silvia Suassuna Carneiro Lúcio

ORCID: https://orcid.org/0000-0002-7639-3635

Faculdade de Ciências Médicas da Paraíba, Brasil E-mail: asilviasuassuna@gmail.com

\begin{abstract}
Resumo
Objetivo: Investigar a prevalência do uso de antidepressivos entre os estudantes da área da saúde no Brasil e os fatores que contribuem para a manutenção desse hábito. Metodologia: Trata-se de uma revisão integrativa da literatura, na qual foram buscados artigos nas bases de dados indexadas na PubMed e na Biblioteca Virtual em Saúde (BVS), dentre elas: LILACS, MEDLINE e BDENF. Foram selecionados, após a análise dos critérios de inclusão e exclusão, uma amostra de 7 artigos. Resultados E Discussão: A maioria dos estudos apresentaram dados relevantes acerca da quantidade de estudantes da área de saúde que utilizam antidepressivos, sendo os Inibidores Seletivos de Recaptação da Serotonina (ISRS) os mais utilizados. Além disso, é apontado que a enorme carga horária, o distanciamento familiar, a dificuldade de fazer novos vínculos de amizade e o pouco tempo para o lazer como fatores motivadores para o consumo desses medicamentos. Conclusão: Frente a essa importante prevalência do uso de medicações antidepressivas pelos universitários da área da saúde no Brasil, infere-se a necessidade de desenvolver medidas de prevenção a fim de minimizar os impactos causados pelos altos níveis de estresse e impedir o desenvolvimento dos sintomas depressivos. Palavras-chave: Estudantes de ciências da saúde; Antidepressivos; Ansiolíticos; Depressão.
\end{abstract}

\begin{abstract}
:
Objective: Investigate the prevalence of the use of antidepressant meds among healthcare students in Brazil and, the factors that contribute to the maintenance of this habit. Methodology: This is an interactive literature review, looking for articles in the databases indexed in PubMed and in the Virtual Health Library (VHL), including: LILACS, MEDLINE and BDENF. After analyzing, by a inclusion and exclusion method, a sample of 7 articles was selected. Results And Discussion: Most studies reveal a relevant data about the number of students in the healthcare area who use antidepressants meds, the Selective Serotonin Reuptake Inhibitors (SSRI) being the most used. Besides, is pointed out that the huge workload, family distance, difficulty to making new friends and the reduced time for leisure are reasons for the use of these medications. Conclusion: Because of this important prevalence of the use of antidepressant medications by university students in the healthcare area in Brazil, is inferred the need of develop preventive measures in order to minimize the impacts caused by high levels of stress and prevent the development of depressive symptoms. Keywords: Students, health occupations; Antidepressive agents; Anxiolytics; Depression.
\end{abstract}




\begin{abstract}
Resumen
Objetivo: Investigar la prevalencia del uso de antidepresivos entre estudiantes de salud en Brasil y los factores que contribuyen al mantenimiento de este hábito. Metodología: Se trata de una revisión integradora de la literatura, en la que se buscaron artículos en las bases de datos indexadas en PubMed y en la Biblioteca Virtual en Salud (BVS), incluyendo: LILACS, MEDLINE y BDENF. Tras analizar los criterios de inclusión y exclusión, se seleccionó una muestra de 7 artículos. Resultados Y Discusión: La mayoría de los estudios presentaron datos relevantes sobre el número de estudiantes del área de la salud que usan antidepresivos, siendo los inhibidores selectivos de la recaptación de serotonina (ISRS) los más utilizados. Además, se señala que la enorme carga de trabajo, la distancia familiar, la dificultad para hacer nuevos lazos de amistad y el poco tiempo para el ocio son factores motivadores para el uso de estos medicamentos. Conclusión: Dada esta importante prevalencia del uso de medicamentos antidepresivos por estudiantes universitarios en el área de la salud en Brasil, se infiere que existe la necesidad de desarrollar medidas preventivas para minimizar los impactos causados por altos niveles de estrés y prevenir el desarrollo de síntomas depresivos.
\end{abstract}

Palabras clave: Estudiantes del área de la salud; Antidepressivos; Ansiolíticos; Depresión.

\title{
1. Introdução
}

A depressão se apresenta como uma das afecções que mais acarreta sofrimento à população, não havendo distinção de gênero, nível socioeconômico ou idade (Carvalho, 2017). A depender da gravidade, esta doença pode tornar uma pessoa incapaz de desempenhar suas atividades de vida diária que até em determinado momento eram tidas como prazerosas, além de causar um sentimento de culpa, baixa autoestima, levando também a escassez de sono ou hipersonia (Barros, 2017; Zuardi, 2017).

Há uma estimativa de que possam existir no mundo, cerca de 300 milhões de pessoas que sofrem com esse mal, levando em consideração ainda, que deste total, em média 10\% chegam a cometer suicídio (taxa anual). Além disso, a depressão é a terceira maior causa de morte entre jovens de 15 a 29 anos no mundo (Penso; Sena, 2020). Já no Brasil, há uma estimativa de que exista cerca de 30 milhões de pessoas que já apresentaram, apresentam ou podem apresentar um quadro depressivo (Carvalho, 2017; Souza, 2020).

Embora não haja predileção por idade, o número de adolescentes e jovens que apresentam esse transtorno é crescente, estando, principalmente, relacionado com o ingresso ao ensino superior (Souza, et al., 2021). Por ser um período de transição complexo, como saída da casa dos pais, novos vínculos de amizade e mudança de cidade, os jovens estão constantemente expostos a situações que demandam tomada de decisões, responsabilidades e cobranças pessoais. Assim, dúvidas, frustrações e ansiedade podem surgir, gerando tristeza e irritabilidade (Barreto, 2020; Brondani et al., 2020).

Nesse contexto, é importante ressaltar que os graduandos de cursos na área da saúde possuem prevalências de depressão e ansiedade bastante aumentadas em relação à população em geral (Gomes; Calixto, 2019). Isso porque estão constantemente expostos à situações estressantes que prejudicam o rendimento acadêmico, provocam o adoecimento mental e colabora com o risco de suicídio. Dessa forma, o comprometimento da saúde mental desses estudantes é preocupante devido aos impactos dessa mazela que, além de causar sofrimento no próprio indivíduo, ainda atrapalha na sua formação profissional e suas relações com os pacientes (Leão et al., 2018).

O tratamento da depressão é feito através da associação entre terapia farmacológica e psicoterapia, entretanto, muitos universitários, geralmente, sem rede de apoio, acabam utilizando apenas a medicação, transformando-a como solução única e imediatista para seus problemas (Dantas et al., 2021). Ademais, a falta de conhecimento sobre os efeitos colaterais, o acesso rápido de informações na internet e a automedicação são fatores que contribuem para o crescimento desse consumo, sendo um problema de saúde pública, já que promove um uso indiscriminado e abusivo, podendo gerar até mesmo uma dependência severa (Pereira; Miceli, 2018).

Dessa forma, a realização de estudos que abordem tal problemática é de grande relevância para o desenvolvimento e articulação de estratégias de prevenção a partir da melhor compreensão acerca das motivações e fatores relacionados com esse 
consumo. Dado o exposto, o presente estudo tem como objetivo investigar a prevalência do uso de antidepressivos entre os estudantes da área da saúde no Brasil bem como os fatores que contribuem com a manutenção desse hábito.

\section{Metodologia}

Esta investigação caracteriza-se como uma revisão integrativa de literatura (RIL). Optou-se por tal método com o objetivo de realizar e organizar um levantamento de dados resultantes de pesquisas a respeito de um tema chave, de maneira clara e objetiva, para proporcionar a construção de um conhecimento científico (Ercole, Melo, Alcoforado, 2014).

Para construção desse artigo de revisão integrativa foram executadas as seguintes etapas: identificação e delimitação do tema; criação da pergunta norteadora e o objetivo da pesquisa; estabelecimento dos critérios de inclusão e exclusão; comparação dos principais resultados; síntese dos principais resultados encontrados.

Para subsidiar a construção do presente estudo, elaborou-se as seguintes questões norteadoras: Qual a prevalência do uso de antidepressivos entre os estudantes da área da saúde no Brasil? Qual a característica das publicações científicas nacionais e internacionais acerca da prevalência do uso de drogas antidepressivas por estudantes da área da saúde do Brasil?

A coleta dos dados foi realizada no mês de março de 2021, mediante a busca em fontes indexadas em bancos de dados da PubMed e da Biblioteca Virtual em Saúde (BVS): Literatura Latino-Americana e do Caribe em Ciências da Saúde (LILACS), Base de Dados em Enfermagem (BDENF) e Medical Literature Analysis and Retrieval System Online (MEDLINE). Os descritores utilizados, a partir da busca em Descritores em Ciências da Saúde (DECS), foram "estudantes de ciências da saúde", "estudantes de medicina", "ansiolíticos", "estudantes de enfermagem", “antidepressivos", "depressão", "uso de medicamentos", "ansiedade", "estudantes", "students, health occupations", “drug”, "medical students", “antidepressants" associados ao operador booleano AND.

A seleção dos artigos na base de dados BVS, foi dividida em partes, nas quais associaram-se os descritores da seguinte forma: (1) "estudantes de medicina" AND "ansiolíticos", na qual foram encontrados um total de 14 artigos, após aplicados os seguintes filtros, texto completo e últimos 5 anos, ficando apenas 3 artigos, dos quais 2 foram excluídos por fugirem do tema proposto; (2) "estudantes de enfermagem" AND “antidepressivos", na qual foram encontrados um total de 7 artigos, sendo 4 artigos excluídos por não serem publicados nos últimos 5 anos, 2 destes restantes não foram selecionados por fuga do tema proposto; (3) "medical students" AND "antidepressants", na qual foram encontrados um total de 2 artigos, os quais os dois serão usados; (4) "estudantes da área da saúde" AND "depressão", possuindo um total de 205 artigos, após a aplicação dos filtros, texto completo, últimos 5 anos, idioma português, ficando um total de 24 artigos, destes foram excluídos 23 artigos, um por duplicidade e 22 por fuga de tema, assim sendo selecionado apenas um estudo; (5) "uso de medicamentos" AND "ansiedade" AND "depressão" AND "estudantes" na qual foram encontrados um total de 34 artigos, após aplicados os seguintes filtros: texto completo, últimos 5 anos e idioma (português, inglês e espanhol), restaram apenas 10 artigos, dos quais 8 foram excluídos por não abordarem o tema adequadamente e um artigo excluído por duplicidade, restando também apenas 1 artigo.

Na base de dados da PubMed associou-se os descritores "health students" AND "drug" na qual foram encontrados um total de 11.649 artigos, após aplicados os seguintes filtros: texto completo, últimos 5 anos e idioma português, restaram apenas 30 artigos, dos quais 29 foram excluídos por não abordarem o tema, sendo apenas 1 estudo selecionado. A estratégia de seleção dos artigos para definição da amostra está resumida no Quadro 1. 
Quadro 1. Identificação, seleção e exclusão dos artigos.

\begin{tabular}{|c|c|c|c|}
\hline \multicolumn{4}{|c|}{ BASES DE DADOS } \\
\hline \multicolumn{4}{|c|}{ BVS } \\
\hline Associação de descritores & Estudos & Critério de exclusão & $\begin{array}{l}\text { Estudos } \\
\text { excluídos }\end{array}$ \\
\hline "estudantes de medicina" AND "ansiolíticos" & 14 & $\begin{array}{l}\text { Ausência de texto completo + publicação } \\
\text { anterior a cinco anos + não relacionado ao tema }\end{array}$ & 13 \\
\hline $\begin{array}{l}\text { "estudantes de enfermagem" AND } \\
\text { "antidepressivos" }\end{array}$ & 7 & $\begin{array}{l}\text { Publicação anterior a cinco anos + não } \\
\text { relacionado ao tema }\end{array}$ & 6 \\
\hline "medical students" AND "antidepressants" & 2 & -- & -- \\
\hline "estudantes da área da saúde” AND “depressão" & 205 & $\begin{array}{l}\text { Ausência de texto completo + publicação } \\
\text { anterior a cinco anos + não relacionado ao tema } \\
\quad+\text { outro idioma +duplicidade }\end{array}$ & 204 \\
\hline $\begin{array}{l}\text { "uso de medicamentos" AND "ansiedade" AND } \\
\text { "depressão" AND “estudantes" }\end{array}$ & 34 & $\begin{array}{l}\text { Ausência de texto completo + publicação } \\
\text { anterior a cinco anos + não relacionado ao tema } \\
+ \text { + outro idioma +duplicidade }\end{array}$ & 33 \\
\hline \multicolumn{4}{|c|}{ PubMed } \\
\hline Associação de descritores & Estudos & Critério de exclusão & $\begin{array}{c}\text { Estudos } \\
\text { excluídos }\end{array}$ \\
\hline \multirow[t]{2}{*}{ "health students" AND “drug" } & \multirow[t]{2}{*}{11.649} & $\begin{array}{l}\text { Ausência de texto completo + publicação } \\
\text { anterior a cinco anos + outro idioma }\end{array}$ & 11.619 \\
\hline & & Não relacionado ao tema & 29 \\
\hline \multicolumn{4}{|c|}{$\begin{array}{l}\text { CRITÉRIOS DE INCLUSÃO: Artigos completos disponíveis eletronicamente, no idioma português, publicados nos últimos } \\
\text { cinco anos, abordando diretamente a temática proposta }\end{array}$} \\
\hline \multicolumn{4}{|c|}{ AMOSTRA : } \\
\hline \multicolumn{4}{|c|}{ BVS (60) + PubMed (1) = 7 ESTUDOS } \\
\hline
\end{tabular}

Fonte: Autores (2021).

\section{Resultados e Discussão}

Após a seleção feita a partir da leitura prévia do título e resumo das fontes elegíveis, a amostra final foi composta por 7 artigos, dos quais serão alocados por meio da Tabela 1, a partir das seguintes variáveis: título da obra, autoria e ano de publicação, base de indexação, tipo de estudo e periódico de publicação. Na sequência, efetuou-se a comparação dos principais resultados com o conhecimento teórico, sendo construídas duas categorias para análise, cujos temas são: (1) Prevalência sobre o uso de antidepressivos por estudantes da área da saúde; (2) Fatores motivadores para o uso de antidepressivos por estudantes da área da saúde, seguindo com a devida síntese das informações.

Após a triagem e seleção dos estudos, a amostra final desta revisão foi constituída por sete publicações, que foram avaliadas levando-se em consideração os autores, o ano de publicação, o título, a base de dados/biblioteca virtual na qual foram encontrados, bem como o periódico de publicação, que versaram sobre a temática "Prevalência do uso de drogas antidepressivas por estudantes da área da saúde do Brasil”. Tal caracterização encontra-se explicitada na Tabela 1.

A partir da análise dos mesmos, constatou-se que os idiomas dos artigos eram em sua maioria em português com 71,4\% e 28,6\% em inglês. No que tange ao ano de publicação, constatou-se que a variação foi de 2017 a 2020, com maior prevalência no ano de 2019 com três $(42,8 \%)$ artigos, seguindo de 2020 que apresentou dois $(28,6 \%)$ e, por fim, os anos de 2018 e 2017 , cada um com apenas um artigo $(14,3 \%)$.

Quanto à base de dados de indexação observou-se que houve predomínio da biblioteca virtual LILACS em relação às demais, possuindo um total de quatro $(57,1 \%)$ artigos, seguida por LILACS/BDENF com dois $(28,6 \%)$ artigos e por último MEDLINE com um (14,3\%) artigo. O periódico encontrado em maior proporção foi a Revista Brasileira de Educação Médica com três $(42,8 \%)$ artigos publicados, seguido pelos seguintes periódicos - Revista da Associação Médica Brasileira, Revista 
Nursing, Revista Portuguesa de Enfermagem de Saúde Mental e International Review of Psychiatry que apresentaram um $(14,3 \%)$ artigo cada uma delas em suas publicações.

Em relação aos tipos de estudos epidemiológicos, observou-se que houve maior prevalência do tipo transversal com três $(42,8 \%)$ artigos, seguido pelos estudos observacional quantitativo e qualitativo, observacional quantitativo, revisão de literatura e revisão integrativa, encontrados no restante da amostra, em um (14,3\%) artigo, respectivamente.

O tema sobre uso de drogas antidepressivas por estudantes da área da saúde, necessita ser bastante debatido, a fim de se discutir e melhorar a assistência e abordagem ofertada aos estudantes nas mais diversas vertentes. Dessa forma, considera-se escasso o número de artigos científicos encontrados com o intuito de responder as perguntas norteadoras desta pesquisa.

Tabela 1. Tabela dos artigos selecionados para o estudo dividida por: autor, ano, idioma, tipo de estudo, periódico de publicação e base de dados.

\begin{tabular}{|c|c|c|c|c|c|}
\hline TÍTULO & $\begin{array}{c}\operatorname{AUTOR}(\mathbf{E S}), \\
\text { ANO }\end{array}$ & IDIOMA & $\begin{array}{l}\text { TIPO DE } \\
\text { ESTUDO }\end{array}$ & $\begin{array}{l}\text { PERIÓDICO DE } \\
\text { PUBLICAÇÃO }\end{array}$ & $\begin{array}{c}\text { BASE DE } \\
\text { DADOS/BIBLI } \\
\text { OTECA }\end{array}$ \\
\hline $\begin{array}{l}\text { Sintomas de depressão, } \\
\text { ansiedade e uso de } \\
\text { medicamentos em } \\
\text { universitários }\end{array}$ & $\begin{array}{l}\text { LELIS, K. C. et } \\
\text { al., } 2020\end{array}$ & Português & Transversal & $\begin{array}{l}\text { Revista Portuguesa de } \\
\text { Enfermagem de Saúde } \\
\text { Mental }\end{array}$ & $\begin{array}{l}\text { LILACS- } \\
\text { Express } \\
\text { BDENF } \\
\text { Enfermagem }\end{array}$ \\
\hline $\begin{array}{c}\text { Tratamento Psíquico } \\
\text { Prévio ao Ingresso na } \\
\text { Universidade: Experiência } \\
\text { de um Serviço de Apoio ao } \\
\text { Estudante }\end{array}$ & $\begin{array}{l}\text { ROCHA, A.M.C. } \\
\text { et. al., } 2020\end{array}$ & Português & $\begin{array}{l}\text { Observacional, } \\
\text { quantitativo }\end{array}$ & $\begin{array}{l}\text { Revista Brasileira de } \\
\text { Educação Médica }\end{array}$ & $\begin{array}{l}\text { LILACS- } \\
\text { Express }\end{array}$ \\
\hline $\begin{array}{c}\text { A depressão como } \\
\text { obstáculo para os futuros } \\
\text { enfermeiros }\end{array}$ & $\begin{array}{c}\text { MORAIS } \\
\text { JUNIOR, S. L. A. } \\
\text { de et al., } 2019\end{array}$ & Português & $\begin{array}{l}\text { Revisão } \\
\text { integrativa }\end{array}$ & Revista Nursing & $\begin{array}{l}\text { LILACS- } \\
\text { Express } \\
\text { BDENF } \\
\text { Enfermagem }\end{array}$ \\
\hline $\begin{array}{c}\text { Prevalência de } \\
\text { Transtornos Mentais } \\
\text { Comuns entre Estudantes } \\
\text { de Medicina da } \\
\text { Universidade Regional de } \\
\text { Blumenau (SC) }\end{array}$ & $\begin{array}{l}\text { GRETHER, E. O. } \\
\text { et. al., } 2019\end{array}$ & Português & Transversal & $\begin{array}{l}\text { Revista de Brasileira de } \\
\text { Educação Médica }\end{array}$ & LILACS \\
\hline $\begin{array}{l}\text { Estressores, sofrimento } \\
\text { psíquico e problemas de } \\
\text { saúde mental entre } \\
\text { estudantes de medicina } \\
\text { brasileiros }\end{array}$ & $\begin{array}{l}\text { CASTALDELLI- } \\
\text { MAIA, J. M. et. } \\
\text { al., } 2019\end{array}$ & Inglês & Transversal & $\begin{array}{c}\text { International Review of } \\
\text { Psychiatry }\end{array}$ & MEDLINE \\
\hline $\begin{array}{c}\text { O uso de drogas e } \\
\text { estudantes de medicina: } \\
\text { uma revisão da literatura }\end{array}$ & $\begin{array}{l}\text { CANDIDO, F. J. } \\
\text { et. al., } 2018\end{array}$ & Inglês & $\begin{array}{l}\text { Revisão da } \\
\text { literatura }\end{array}$ & $\begin{array}{l}\text { Revista da Associação } \\
\text { Médica Brasileira }\end{array}$ & $\begin{array}{l}\text { LILACS- } \\
\text { Express }\end{array}$ \\
\hline $\begin{array}{l}\text { Análise da Depressão, dos } \\
\text { Fatores de Risco para } \\
\text { Sintomas Depressivos e do } \\
\text { Uso de Antidepressivos } \\
\text { entre Acadêmicos do } \\
\text { Curso de Medicina da } \\
\text { Universidade Estadual de } \\
\text { Ponta Grossa }\end{array}$ & $\begin{array}{l}\text { CYBULSKII, C. } \\
\text { A.; MANSANII, } \\
\text { F. P., } 2017\end{array}$ & Português & $\begin{array}{l}\text { Observacional, } \\
\text { quantitativo, } \\
\text { qualitativo }\end{array}$ & $\begin{array}{l}\text { Revista Brasileira de } \\
\text { Educação Médica }\end{array}$ & $\begin{array}{l}\text { LILACS- } \\
\text { Express }\end{array}$ \\
\hline
\end{tabular}




\section{Discussão}

\subsection{Prevalência sobre o uso dos antidepressivos por estudantes da área da saúde}

A maioria dos estudos apresentaram uma prevalência significativa sobre o uso de medicamentos antidepressivos entre estudantes de cursos da área da saúde no Brasil. Em uma universidade estadual do Paraná, foi visto que 29,15\% dos alunos de medicina usam ou usaram esses medicamentos e 18,09\% estavam usando durante o período da pesquisa (Cybulskii, Mansanii, 2017). Dado semelhante foi encontrado nesta mesma população em uma universidade pública de Santa Catarina, em que 19,2\% usavam drogas antidepressivas (Grether et al., 2019).

Um dos estudos de revisão integrativa apontou uma prevalência de $26 \%$ do uso de antidepressivos entre graduandos de enfermagem de uma universidade pública no interior de Minas Gerais (Morais Junior et al., 2019). Em relação ao uso desses medicamentos, outra revisão apresentou uma estatística de 11, 4\% em estudantes de medicina no Brasil (Candido et al., 2018). Num estudo realizado com estudantes de medicina em quatro universidades do estado de São Paulo, 15,5\% relataram uso prévio de antidepressivos, enquanto que 11,6\% admitiram o uso atual de antidepressivos (Castaldelli-Maia et al., 2019).

$\mathrm{Na}$ pesquisa feita com os ingressados nos cursos de medicina, fonoaudiologia e tecnologia de radiologia numa universidade de Minas Gerais foi relatado que 42,5\% dos ingressados já fizeram uso de drogas psicoativas (Rocha et al., 2020). Nesse mesmo estado, em uma instituição privada com estudantes da área da saúde, 5,3\% dos entrevistados usam frequentemente antidepressivos, enquanto 13,3\% raramente utilizavam (Lelis et.al., 2020).

Em relação ao sexo, estudo relata que as mulheres apresentam um maior consumo de antidepressivos e ansiolíticos enquanto os homens têm maior tendência de consumo de outros tipos de medicamentos (Candido et al., 2018). Observou-se que os Inibidores Seletivos de Recaptação da Serotonina (ISRS) foi a classe de antidepressivos mais utilizada pelos universitários da área da saúde (Cybulskii, Mansanii, 2017; Morais Junior et al., 2019; Rocha et al., 2020). Segundo Cybulskii e Mansanii (2017), o escitalopram foi o mais usado, enquanto que Morais Júnior et al. (2019) encontrou uma maior frequência de uso de cloridrato de fluoxetina (63\%), seguido pelo cloridrato de amitriptilina (antidepressivo tricíclico) e cloridrato de paroxetina (ISRS).

Nesse estudo foi visto também que a maioria dos alunos de medicina usuários de antidepressivos apresentavam baixa adesão ao tratamento, sendo apontado apenas 33,3\% desses usuários como alto grau de adesão, o que é preocupante devido à maior chance de agravamento da doença e maior redução da função cognitiva. Além disso, foi apontado que esses estudantes não estão recebendo tratamento adequado para seus transtornos depressivos, já que há uma baixa adesão ao tratamento medicamentoso frente a uma alta prevalência dos sintomas depressivos e a um escore maior ou igual a 11 na Escala de depressão de Beck nos usuários de antidepressivos (Cybulskii, Mansanii, 2017).

\subsection{Fatores motivadores para o uso de antidepressivos por estudantes da área da saúde}

Segundo Grether et al. (2019), o maior uso de antidepressivos e ansiolíticos está relacionado com o Transtorno Mental Comum (TCM), embora 28,6\% dos graduandos de medicina entrevistados usassem essas drogas mesmo sem terem o transtorno. Ademais, concluiu-se que estudantes com cargas horárias acadêmicas diárias maiores que 8 horas, com menos de 2 horas por dia de lazer e os que nunca cogitaram ou não cogitam mais o abandono do curso são os que proporcionalmente apresentaram maior frequência de TCM. Isso porque esses mesmos alunos mostravam comportamento mais competitivo, insatisfação com a extensa carga horária e pequeno tempo de lazer, tinham dificuldade em fazer novos amigos, sentiam falta de apoio emocional e até mesmo relataram rejeição pelos amigos da mesma faixa etária.

A partir disso, resultados semelhantes foram encontrados em outra pesquisa, afirmando que a própria grade curricular do curso de medicina contribui fortemente para o desenvolvimento de sintomas depressivos nos estudantes, em especial no final da graduação (Cybulskii, Mansanii, 2017). No entanto, o estudo de Rocha et al (2020) apontou uma maior prevalência do uso de antidepressivos em estudantes dos dois primeiros anos do curso $(34,5 \%)$. Supõe-se que isso se deve a necessidade de adaptação 
do estudante ao ambiente acadêmico, aumento das responsabilidades e estabelecimento de novos vínculos. O estudo ainda mostra que os acadêmicos de Medicina, têm maior risco de desenvolverem sintomas depressivos e utilizarem os psicofármacos, quando comparados aos demais estudantes da área da saúde.

$\mathrm{Na}$ revisão da literatura, também foram mencionados como fatores estressantes: a enorme carga horária, a privação familiar e social e o enfrentamento das situações de morte e sofrimento (Candido et al., 2018). Em outro estudo, os motivos encontrados para o uso de antidepressivos entre graduandos de enfermagem foram: insônia, fadiga, depressão, síndrome do pânico, falta de concentração e tristeza (Morais Junior et al., 2019).

\section{Conclusão}

Portanto, os estudos apontam relevante prevalência de uso de medicações antidepressivas entre os universitários da área da saúde no Brasil, estando ligado aos altos níveis de estresse e desenvolvimento de sintomas depressivos que são provocados pela carga horária exaustiva, fragilidade na rede de apoio familiar e social, redução dos momentos de lazer e frequente vivência de momentos de morte e sofrimento.

Além disso, é importante ressaltar a problemática envolvendo os graduandos de medicina que sofrem riscos de desenvolverem transtornos depressivos e de ansiedade devido à própria grade curricular, sendo necessária uma articulação de estratégias de prevenção pelas instituições a fim de minimizar esses impactos na saúde biopsicossocial e espiritual desses alunos.

Por fim, reconhecemos as limitações deste estudo, como a utilização de pesquisas realizadas nas regiões Sudeste e Sul, e recomendamos a realização de estudos nessa temática nas outras regiões do Brasil, principalmente, no Centro-oeste e Norte, além de ser preciso o fomento de pesquisas longitudinais que acompanhem esses estudantes ao longo do tempo para que possam ajudá-los no desenvolvimento de medidas preventivas e tratamentos precoces.

\section{Referências}

Barreto, S. (2020). Depressão em jovens universitários. Revista Enfermagem Contemporânea, 9(1), 6-8. https://www5.bahiana.edu.br/index.php/enfermage $\mathrm{m} /$ article/view/2852

Barros, M. B. A. et al. (2017). Depressão e comportamentos em saúde em adultos brasileiros - PNS 2013. Revista Saúde Pública. 51(1), 1-8. https://www.scielo.br/j/rsp/a/rJgc4vNn6tKZXqTSvV5DXMb/?lang=pt\&format=pdf

Brondani, M. A. et al. (2019) Depressão em estudantes universitários: fatores de risco e protetivos e sua relação nesse contexto. Disciplinarum Scientia. 20(1), 137-149. https://periodicos.ufn.edu.br/index.php/disciplinarumS/article/view/2629

Candido, F. J. et al. (2017). The use of drugs and medical students: a literature review. Rev. Assoc. Med. Bras., 64(5), 462-468. https://www.scielo.br/j/ramb/a/FtkbW9bw5KW6mDzzFCRCqFc/?format=pdf\&lang=en

Carvalho, M. C. P. et al. (2017). Levantamento da situação de saúde mental e uso de ansiolíticos e antidepressivos por acadêmicos do curso de odontologia de uma universidade do Sul de Minas Gerais. Revista da Universidade Vale do Rio Verde 15(1), 489- 496. http://periodicos.unincor.br/index.php/revistaunincor/article/view/2772

Castaldelli-Maia, J. M. et al. (2019). Estressores, sofrimento psíquico e problemas de saúde mental entre estudantes de medicina brasileiros. International Review of Psychiatry. 31(7), 603-607. https://www.tandfonline.com/doi/full/10.1080/09540261.2019.1669335

Cybulski, C. A. \& Mansani, F. P. (2017). Análise da depressão, dos fatores de risco para sintomas depressivos e do uso de antidepressivos entre acadêmicos do curso de medicina da Universidade Estadual de Ponta Grossa. Rev. bras. educ. med. 41(1), 92-101. https://www.scielo.br/j/rbem/a/qZQbVnkyfT9pPQP3qqSPQHw/?lang=pt\&format=pdf

Dantas, R. C. O. et al. (2021) Uso de antidepressivos por universitários: Revisão integrativa. In: NASCIMENTO, A.R.S. et al. Saúde Mental e Suas Interfaces: Rompendo Paradigmas, 10-17. https://www.poisson.com.br/livros/individuais/Saude_Mental_Paradigmas/Saude_Mental_Paradigmas.pdf\#page=10

Ercole, F. F., Melo, L. S. \& Alcoforado, C. L. G. C. (2014) Revisão integrativa versus sistemática. Rev. Min.Enferm. 18(1), 12-14. https://cdn.publisher.gn1.link/reme.org.br/pdf/v18n1a01.pdf

Gomes, L. F., Calixto, M. F. (2019). Saúde mental e ensino superior: A relação da universidade com o surgimento e/ou agravo de sofrimento mental em estudantes universitários. Trabalho de conclusão de curso (TCC) em graduação de enfermagem. https://ri.cesmac.edu.br/bitstream/ted e/561/1/Saúde\%20mental\%20e\%20ensino\%20superio\%20-\%20a\%20relação\%20da\%20universidade\%20com\%20o\%20surgimento\%20eou\%20agravo\%20de $\% 20$ s ofriment o\%20mental $\% 20$ em\%20estudantes\%20universitários..pdf 
Research, Society and Development, v. 10, n. 11, e286101119641, 2021

(CC BY 4.0) | ISSN 2525-3409 | DOI: http://dx.doi.org/10.33448/rsd-v10i11.19641

Grether, E. O. et al. (2019). Prevalência de transtornos mentais comuns entre estudantes de edicina da Universidade Regional de Blumenau (SC). Rev. bras. educ. med., 43(1), 276-285. https://www.scielo.br/j/rbem/a/SjhFWSSNjFCMrGn9qwqrq4P/?lang=pt

Leão, A. M. et al. (2018). Prevalência e fatores associados à depressão e ansiedade entre estudantes universitários da área da saúde de um grande centro urbano do Nordeste do Brasil. Rev. bras. educ. med. 42(4), 55-65. https://www.scielo.br/j/rbem/a/kyYq35bwkZKHpKRTjyqjMYz/?lang=pt\&format=html

Lelis, K. C. G. et al. (2020). Sintomas de depressão, ansiedade e uso de medicamentos em universitários. Revista Portuguesa de Enfermagem de Saúde Mental. 23(1), 9-14. https://scielo.pt/scielo.php?script=sci_arttext\&pid=S1647-21602020000100002?script=sci_arttext\&pid=S1647-21602020000100002

Morais Junior, S. L. A. et al. (2019). A depressão como obstáculo para os futuros enfermeiros. Revista Nursing. 22(253), 2973-2978. http://www.revistanursing.com.br/revistas/253/pg81.pdf

Penso, M. A. \& Sena, D. P. A. A. (2020). Desesperança do jovem e o suicídio como solução. Soc. estado. 35(1), 61-81. https://www.scielo.br/j/se/a/rLfXhwgd7qgpBzMSrjwFXmj/?lang=pt

Pereira, R. E. \& MICELI, B. C. (2018). Fatores que influenciam o consumo de antidepressivos em universitários dos cursos de saúde de uma instituição de ensino superior privada do município de Sete Lagoas - MG. Revista Brasileira de Ciências da Vida, 6(4), 113-128. http://jornalold.faculdadecienciasdavida.com.br/index.php/RBCV/article/view/882/457

Rocha, A. M. C. et al. (2020). Tratamento psíquico prévio ao ingresso na universidade: experiência de um serviço de apoio ao estudante. Rev. bras. educ. med 44(3), 1-7. https://www.scielo.br/j/rbem/a/mXDJ8FDpbJQmn5XQzcDcDNy/?lang=pt

Souza, R. C. et al. (2021). O uso de antidepressivos em estudantes da área da saúde. Brazilian Journal of Development, 7(4), 40842-40852. https://www.brazilianjournals.com/index.php/BRJD/article/view/28588/22577

Souza, M. S. P. et. al, (2021). Uso de antidepressivos e ansiolíticos por alunos do curso de farmácia de uma instituição pública e privada do interior da Bahia. Research, Society and Development. 10(8), 1-8. https://rsdjournal.org/index.php/rsd/article/view/17177

Zuardi, A. W. (2017). Características básicas do transtorno de ansiedade generalizada. Medicina (Ribeirão Preto), 50(1), 51-55. https://www.revistas.usp.br/rmrp/article/view/127538/124632 